\title{
Performance Comparison of Distance and Density based Clustering Algorithm (DDCSA) V/s Normal Technique to analyze Power Consumption and Network Lifetime of Wireless Sensor Networks
}

\author{
Naveen Sharma ${ }^{\dot{A}}$, Malti Rani ${ }^{\dot{A}}$ and Anand Nayyar ${ }^{\dot{B}^{*}}$ \\ ${ }^{\dot{A}}$ Department of Computer Science, Punjab Technical University, India \\ ${ }^{\dot{B}}$ Department of Computer Applications \& IT, KCL Institute of Management \& Technology, India
}

Accepted 10 May 2014, Available online 01 June 2014, Vol.4, No.3 (June 2014)

\begin{abstract}
Aggregating data of several nodes in a wireless sensor networks allows us to accurately monitor a remote environment. However, low-cost battery-powered nodes are often used to implement such networks, resulting in tight energy and communication bandwidth constraints. We have found that sensor node distances and densities are key factors in clustering. The aim of this paper is to introduce a new protocol to reduce overall power consumption, extend network life time in wireless sensor networks. In this paper, Normal Technique and Distance and Density based Clustering Algorithm (DDCSA) technique is compared on various parameters. Simulation results show a better performance of DDCSA as compared to Normal Technique
\end{abstract}

Keywords: Distance, Cluster Head (CH), Density, Data Aggregation, Wireless Sensor Networks

\section{Introduction}

A Sensor networks is a wireless network consisting of spatially distributed autonomous devices using sensors to cooperatively monitor environmental conditions (S. Bandyopadhyay et al, 2003). Wireless sensor networks (WSNs) are composed of large number of low-cost and tiny sensors. It is a distributed and self-organized network where sensor nodes will locally carry out sensing, processing and transmitting operations in an autonomous and unattended manner. WSNs have broad applications such as military surveillance and tracking, environment monitoring and forecasting, healthcare etc (N. Sharma et $a l, 2014$; K. Akkaya et al, 2005). Clustering is very much useful in those cases where sensor nodes are densely deployed causing them to detect common phenomena leading to high redundancy in their sensed raw data. Thus reporting raw data is often unnecessary. The data aggregation protocols designed for WSNs exploits the naturally existing redundancy and computes the aggregate data, thereby reducing the communication cost and energy expenditure in data collection. During a typical data aggregation process, sensor nodes are organized into groups forming a hierarchical structure with the root at BS. Within the hierarchical structure, the non-leaf nodes act as aggregators or cluster heads and others as members. The leaf nodes send their raw data to their immediate aggregator which fuses the data collected from its children and forwards the fusion result to BS.

*Corresponding author: Anand Nayyar

DOI: http://Dx.Doi.Org/10.14741//jcet/2014.4.3.64
In this way, data are processed and fused at each hop on the way to the BS, and communication overhead is greatly reduced (N. Sharma et al, 2014). We divide existed strategies into two categories. In single-input coding each node can consider data from only one other source during data compression (P. V. Rickenbach et al, 2004; R. Cristescu et al, 2005). In multi-input coding strategies aggregation exploits correlation in data from multiple sources.

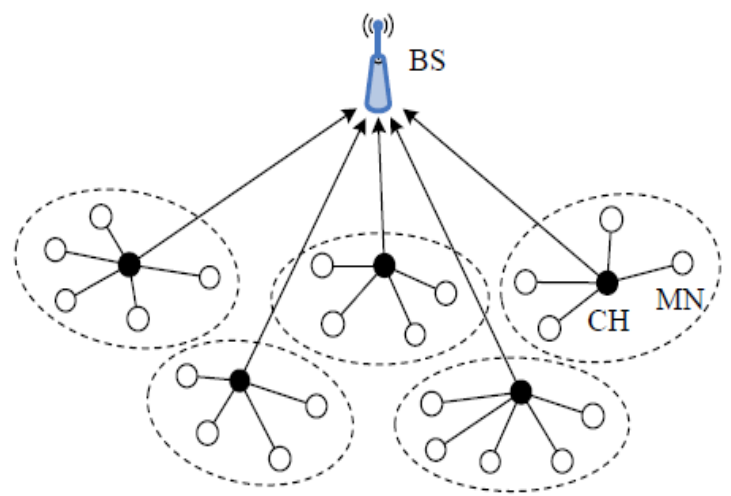

Fig.1 Data Aggregation in WSN

Multi-input coding strategies are generally based on clusters of WSN nodes. So we call them cluster compression. Cluster-based routing schemes data aggregation is done in cluster-heads only, while in singeinput routing schemes every intermediate node can performs data aggregation. This property generally reduces the communication and computation costs of 
cluster compression (A. Goel et al, 2005; F. Ye et al, 2003).

\section{Literature Review}

Recently, various clustering technique to reduce an energy consumption of sensor nodes have been developed. One of the most well-known clustering approaches is LEACH (Low- Energy Adaptive Clustering Hierarchy) a clustering-based protocol that utilizes a randomized rotation of local cluster head to evenly distribute the energy load among the sensors in the network. But, since node has different communication distance and remainder energy, LEACH cannot have chance to cluster head and consume their energy evenly with only probability. In addition, when the cluster head select, the cluster head causes on additional overhead to estimate the sum of the current energy (W. Heinzelman et al, 2014).

PEGASIS is an extension of LEACH, in which all nodes are organized into a chain and each node transmits data only to its neighbor. However, neither LEACH nor PEGASIS optimize cluster sizes to minimize energy consumptions considering spatial correlation (S. Lindsey et al, 2002).

A hybrid energy-efficient distributed (HEED) clustering protocol considers node's residual energy and the cost of communication within the cluster during $\mathrm{CHs}$ selection. It can not only minimize the control overhead, but also prolong network lifetime since $\mathrm{CHs}$ are well distributed (O. Younis et al, 2004).

The author introduced an adaptive decentralized reclustering protocol (ADRP). In ADRP, CHs and next heads are elected on residual energy of each node and the average energy of each cluster (F. Bajaber et al, 2009).

To enhance lifetime, the author proposed an energy efficient clustering protocol (EECPL) which organizes sensors into clusters and uses ring topology to send data packets (F. bajaber et al, 2010).

Density-based Energy-efficient Clustering Algorithm (DECA) ensures the even distribution of cluster heads due to the evaluation of density. Moreover, the residual energy of each cluster head is under consideration after each round of cluster head selection (Zhanyang Xu et al, 2013). Density-based Energy-efficient Game-theoretic Routing Algorithm (DEGRA) is a hierarchical routing algorithm which adopts clustering and ensures even distribution of cluster heads due to the evaluation of nodes' density (Zhanyang Xu, Yue et al, 2012).

\section{Motivation}

This section provides the motivation of DDCSA and Normal techniques. We will discuss the relationship of Data gathering costs, inter cluster cost and intra cluster cost.

\subsection{Intra and Inter Cluster Cost}

The total cluster communication energy is composed of two parts. The intra-cluster cost is the energy consumed by all data communication from the member nodes to the cluster-head in the cluster, which aggregate data from other nodes in a cluster. The inter-cluster cost is the energy consumed by communication between the cluster-head and the sink node. If the entire WSN is composed of one large cluster, there is no inter-cluster cost but the intracluster cost is extremely high. On other extreme, each cluster contains only a single node, for which the intercluster cost is high but the intra-cluster cost is low.

\subsection{Data Gathering Cost}

In cluster-based data gathering, each cluster's communication costs can be divided into intra-cluster and inter-cluster costs. We denote the intra-cluster cost as $\mathrm{C}_{\text {intra,k}}$. A tree $\mathrm{T}_{\mathrm{k}}$ is used to collect data from cluster k. $\alpha$ and $\beta$ are constants indicating the distance-independent and distance-dependent energy components for one-bit communication. According to Equation 1, $\mathrm{C}_{\mathrm{intra}, \mathrm{k}}$ is calculated as follows :

$C_{\text {intra,k }}=\sum_{e_{i} \in T_{k}} E\left(d_{i}\right)=\sum_{e_{i} \in T_{k}} p_{i} \cdot\left(\alpha+\beta d_{i}^{n}\right)$

where di is the transmission distance on the edge $e_{i}$, and $p_{i}$ is the number of bits transmitted on $\mathrm{e}_{\mathrm{i}}$.

The inter-cluster cost $\mathrm{C}_{\text {inter, } \mathrm{k}}$ of cluster $\mathrm{k}$ is defined as the energy consumed by transmitting compressed data from the cluster-head to the sink via route $\mathrm{R}_{\mathrm{k}}$. Equation 2 can be used to estimate the size of compressed data $B_{S}^{k}$ in an s-node cluster $\mathrm{k}$. Therefore, we can calculate $\mathrm{C}_{\mathrm{inter}, \mathrm{k}}$ based on Equation 2.

$C_{\text {inter }, k}=\sum_{e_{i} \in R_{k}} E\left(d_{i}\right)=\sum_{e_{i} \in R_{k}} B_{S}^{k} \cdot\left(\alpha+\beta d_{i}^{n}\right)$

Finally, we get the total transmission cost of the network

$\mathrm{C}_{\text {network }}=\sum K\left(\mathrm{C}_{\text {intra, }}, \mathrm{k}+\mathrm{C}_{\text {inter, } \mathrm{k}}\right)$

by summing all the cluster's communication costs(Heng(-yu Long et al, 2009).

\section{Distance and Density Based Clustering Selection Algorithm (DDCSA)}

Distance and density based clustering technique is an adaptive clustering scheme. If the distance of a region from the sink is large, the optimal cluster size is also large because any data transmitted from this point must be repeatedly retransmitted before reaching to the sink. The inverse is true for clusters near the sink node: smaller clusters are better in this case. In dense regions, large clusters are better because high spatial data correlation leads to a good compression ratio. So, distance and density are two key factors in clustering. The clustering flow can be divided into three steps: (1) select a region for clustering; (2) decide the optimal cluster size for a region; (3) form the cluster by absorbing other neighbor nodes and repeat the process. We start clustering in the regions farthest from the sink. At the beginning of each clustering progress, the farthest unclustered node from sink is selected as the first node in the cluster. We use its distance " $\mathrm{d}$ " to the sink as the reference to calculate the optimal 
cluster size. The cluster's nearest unclustered neighbor nodes are absorbed as members in the cluster. Once the optimal cluster size is reached, the process is terminated and another clustering process starts, until all nodes belong to clusters. Cluster-head selection for each cluster is based on the shortest distance metric.

\section{Normal Technique}

In Normal technique the sensing region is divided into clusters. Every cluster has a Cluster head. Cluster head selection is based on node's energy and transmission range to destination. If a node has maximum energy but it have not that much transmission range to the destination, then that node will not elected as cluster head. So, only those nodes can become cluster head which has maximum energy and maximum transmission range to the destination. After cluster head selection, an inter and intra cluster routing is done using ad-hoc on demand distance vector routing. In this the routes remain active only as long as data packets are traveling along the paths from the source to the destination. When the source nodes stop sending packets, than the path will time out and close. The cluster heads are changed after every round. In every round only that node will become cluster head which have the maximum energy and maximum transmission range to the destination.

\section{Experimental Setup}

The performance of the Distance and density based cluster selection algorithm (DDCSA) and Normal technique is analyzed against parameters such as throughput, delay, and packet delivery ratio and energy consumption. We choose the total transmission cost per cycle to analyze the performance of DDCSA and compare it with Normal Technique. The simulations are carried out under NS2 simulator running in Linux environment.

Table 1 Simulation Parameters

\begin{tabular}{|l|l|}
\hline Parameter Name & Parameters \\
\hline Channel Type & Channel/ wireless \\
\hline RP Model & Propagation/Two Ray Ground \\
\hline LLtype & LL \\
\hline IQ Type & Queue/Drop tail/PriQueue \\
\hline Max pckt in Ifq & 200 \\
\hline Network Interface Type & Phy/Wireless Phy \\
\hline MAC type & Mac/802-11 \\
\hline No of mobile nodes & 51 \\
\hline Routing protocol & AODV and DDCSA \\
\hline Grid Size & 500 X 500 \\
\hline
\end{tabular}

The main objective of this scheme is to minimize energy consumption and to increase throughput. To analyze the performance of both techniques discussed earlier, scenarios are set as per the parameters shown in the Table 1 . This scenario is carried out in a flat-grid range of $500 \mathrm{X}$ 500 .

\section{Performance Metrics}

The performance of both techniques gets calculated under following metrics.

- Throughput: Throughput provide the vision of data transferring i.e. it refers that in a particular period of time how much packets can be transferred from source to destination.

- Delay: Delay referred as the time difference between the data packet sending at sender's end and the data packet receiving at receiver's end. It shows the average delay of all successfully transmitted packets from source to destination.

- Energy consumption: It describe as the total energy consumption to the number of packets received by the sink (receiver) successfully. The energy consumption per data packet received by the sink (receiver) provides the energy efficiency of the protocols.

- Packet Delivery Ratio (PDR): The packet delivery ratio is the ratio of the number of packets delivered to the destination.

\section{Simulation Results and Performance Analysis}

In this paper we analyze the DDCSA and Normal Technique. NS2 is used to simulate the network. Simulation results show a better performance for DDCSA as compared to Normal Technique. As figures 4-7 indicate the lifetime of the network is extended with respect to DDCSA.

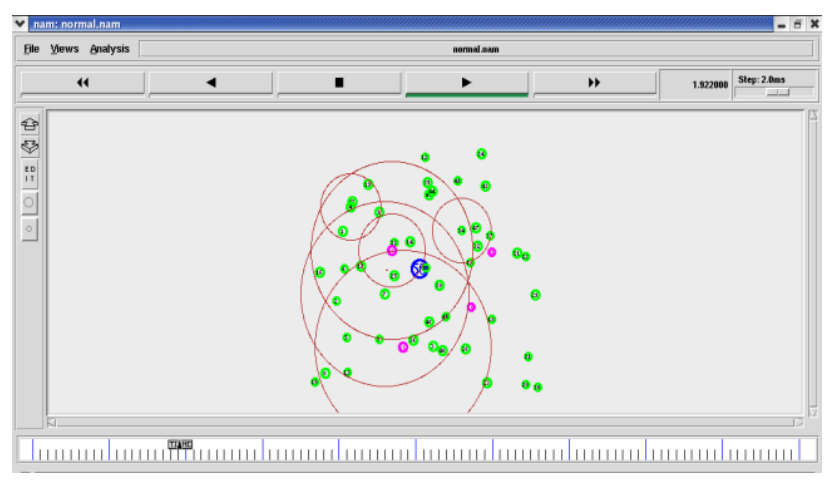

Fig.2 Packet Forwarding using Normal Technique

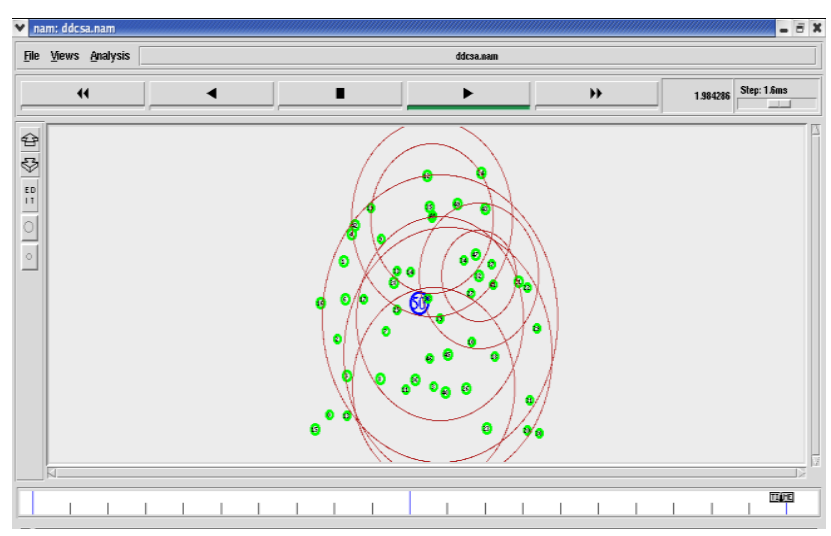

Fig.3 Packet Forwarding using DDCSA Technique

In this scenario (figure 2) we took 51 mobile nodes, make clusters of nodes and data transmission is done using 
AODV routing protocol. The green colored nodes act as member nodes and magenta colored nodes act as cluster heads of each cluster.

In this scenario (figure 3) we took 51 mobile nodes, adaptive clustering is being done by distance and density based clustering (DDCSA) algorithm. In both figures (2 and 3) packet forwarding between nodes has been carried out. In these figures data transferring between nodes is shown and packet loss from the nodes get calculated.

In scenario (figure $3 \& 4$ ) red circles are the ranges of sensor nodes which indicate the range of each node. All the nodes can send their data within that range only. In Normal technique cluster heads are chosen on the basis of node's maximum transmission power. Blue node shows the base station that all nodes have to send the data or we can say that it is the destination for all nodes. Above scenario uses flat grid of size $500 * 500$.

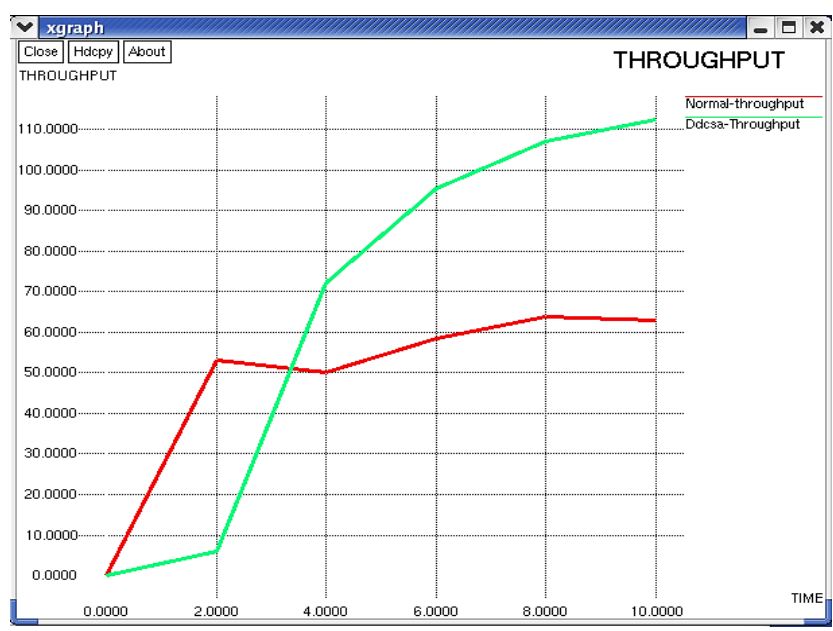

Fig.4 Throughput graph

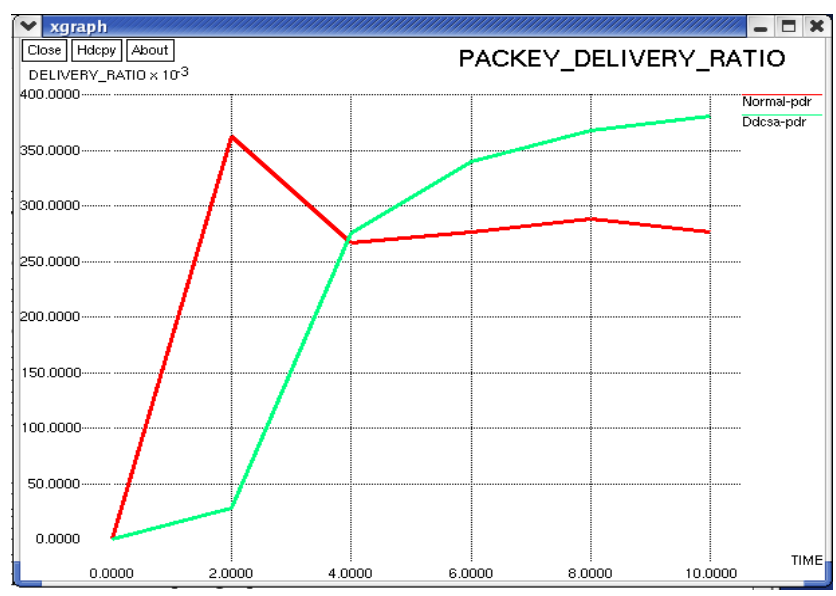

Fig.5 Packet Delivery Ratio

In these graphs (from figure 4-7) compare and analyze the performance of Normal Technique V/s DDCSA by considering parameters like energy, delay, throughput and packet delivery ratio etc. Here comparison shows in form of lines, where red line shows Normal Technique and green line shows DDCSA. It is shown in graphs clearly that DDCSA is better than Normal Technique in all four parameters.

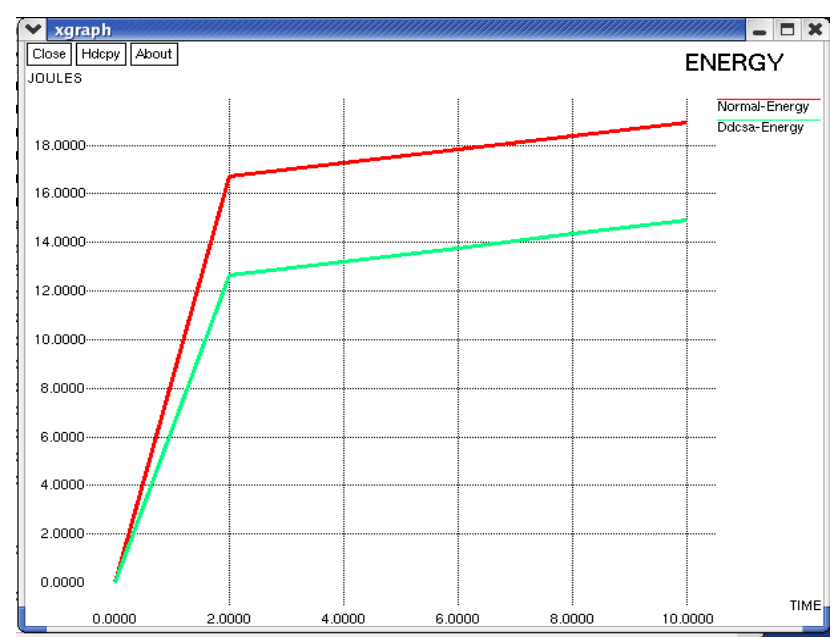

Fig.6 Energy consumption graph

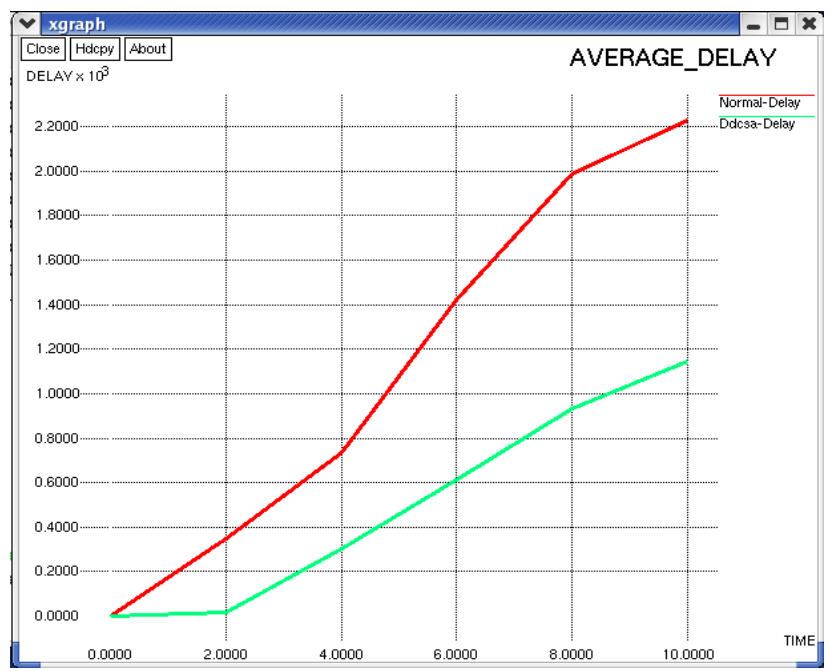

Fig.7 Delay graph

\section{Comparison of DDCSA and Normal Technique}

Table 2 DDCSA and Normal Technique

\begin{tabular}{|l|l|l|}
\hline Parameters & Normal Technique & DDCSA \\
\hline Delay & More & Less \\
\hline Throughput & Low & High \\
\hline Packet Delivery Ratio & Less & More \\
\hline Energy Spent & More & Less \\
\hline
\end{tabular}

\section{Conclusion and Future scope}

Although wireless sensors networks are very popular and vast area of research with growing set of research results, they are limited by the power available through their life cycle. Once their power is depleted, sensors might be replaced or recharged if possible. In this paper we presented two techniques and analyze them on many parameters. Simulation results shows that Distance and density based cluster selection algorithm (DDCSA) outperforms Normal technique and increase the lifetime of the network. In future researcher can enhance DDCSA by using various routing technique and can get better results. 


\section{References}

S. Bandyopadhya, E.J. Coyle (2003), An energy efficient hierarchical clustering algorithm for wireless sensor networks, INFOCOM, vol.3, pp.1713-1723

N. Sharma, A. Nayyar (2014), A Comprehensive Review of Cluster Based Energy Efficient Routing Protocols for Wireless Sensor Networks, International Journal of Application or Innovation in Engineering and Management, vol.3, pp. 442453.

K.Akkaya, M.Younis (2005), A survey on routing protocols in wireless sensor networks, Elsevier Ad Hoc Network, vol.3, no.3, pp.325-349.

P. V. Rickenbach, R. Wattenhofer (2004), Gathering correlated data in sensor networks, Proc. Joint Wkshp. Foundations of Mobile Computing, pp. 60-66.

R. Cristescu, B. Beferull-Lozano, M. Vetterli (2005), Networked Slepian-Wolf: theory, algorithms, and scaling laws, IEEE Trans. Information Theory, vol. 51, pp. 4057-4073.

A. Goel, D. Estrin (2005), Simultaneous optimization for concave costs: Single sink aggregation or single source buy-atbulk, Algorithmica, vol. 43, pp. 5-15.

F. Ye, G. Zhong, J. Cheng, S. Lu, L. Zhang (2003), PEAS: A robust energy conserving protocol for long-lived sensor networks, Proc. Int. Conf. Distributed Computing Systems, pp. 28-37.

N. Sharma, A. Nayyar (2014), A Comprehensive Review of Distance and Density Based Cluster Head Selection Schemes, International Journal of Computer Science and Mobile Computing, vol.3, pp. 204-211.
S. Lindsey, C. S. Raghavendra (2002), PEGASIS: Powerefficient gathering in sensor information systems, Proc. Aerospace Conf., vol. 3, pp. 1125-1130.

O.Younis, S.Fahmy (2004), HEED: A hybrid, energy-efficient, distributed clustering approach for ad hoc sensor networks, IEEE Trans. on Mobile Computing. vol.3, no.4, pp.366-379.

F. Bajaber, I. Awan (2009), Adaptive decentralized re-clustering protocol for wireless sensor networks, Journal of Computer and System Sciences.

F. bajaber, I. Awan (2010), Energy efficient clustering protocol to enhance lifetime of wireless sensor network, J Ambient Intell Human Comput, pp.239-248.

Zhanyang Xu, Yue Yin, Jin Wang (2013), A Density-based Energy-efficient Clustering Algorithm for Wireless Sensor Networks, International Journal of Future Generation Communication and Networking, vol.6.

Zhanyang Xu, Yue Yin, Jin Wang (2012), An Density-based Energy-efficient Routing Algorithm in Wireless Sensor Networks using Game Theory, International Journal of Future Generation Communication and Networking, vol.5.

Hengyu Long, Yongpan Liu, Xiaoguang Fan, Robert P. Dick, Huazhong Yang (2009), Energy-Efficient Spatially-Adaptive Clustering and Routing in Wireless Sensor Networks, IEEE.

W. Heinzelman, A.Chandrakasan, H. Balakrishnan (2000), Energy-efficient communication protocol for wireless sensor networks, Proc. Hawaii International Conference System Sciences, pp.1-10. 